2

\title{
The Wsp intermembrane complex mediates metabolic control of the swim-attach decision of Pseudomonas putida
}

\author{
by
}

Ángeles Hueso-Gil, Belén Calles and Víctor de Lorenzo*

Systems Biology Program, Centro Nacional de Biotecnología-CSIC, Campus de Cantoblanco, Madrid 28049, Spain.

Running Title: $\quad$ The Wsp complex of Pseudomonas putida

Keywords: Pseudomonas putida, Biofilm, cyclic-di-GMP, surface recognition, flagella

\footnotetext{
* Correspondence to: Víctor de Lorenzo
}

Centro Nacional de Biotecnologia, CSIC C/ Darwin, 3

Campus UAM Cantoblanco

Madrid 28049, Spain

Email: vdlorenzo@cnb.csic.es

Pho +34 91-585 4536, Fax +34 91-585 4506 


\section{SUMMARY}

Pseudomonas putida KT2440, a microorganism of interest for biotechnological purposes, is one amongst the many bacteria that attach to surfaces and produce biofilm. Although other mechanisms that contribute to this decision have been studied until now, a 7-genes-operon with a disposition and homology shared with the wsp operon in Pseudomonas aeruginosa remained to be investigated. In this work, we characterized the function of $P$. putida wsp operon by the combination of deletion mutants with complementations with $P$. aeruginosa's genes and with deletions of 3 other genes: the genes that code for the transcription factors fle $Q$ and fleN and the flagellar movement regulator, fglZ. Examining mutant behaviour at 6 and 24 hours under three different carbon regimes (citrate, glucose and fructose) we saw that this complex carries out a similar function in both Pseudomonas. In P. putida, the key components are WspR, a protein that harbours the domain for producing c-di-GMP, and WspF, which controls its activity. Transformation with the equivalent proteins of $P$. aeruginosa had a significant impact on of $P$. putida mutant phenotypes and could complement their functions under some conditions. These results contribute to the deeper understanding of the complex element network that regulate lifestyle decision in P. putida

\section{INTRODUCTION}

The environmental bacterium Pseudomonas putida KT2440 has is growingly receiving attention a a suitable chassis for a suite or biotechnological and environmental applications. In this context, the characterization of the mechanisms that trigger the swim or attach decision are decisive for e.g. developing catalytic biofilms. Its close relative Pseudomonas aeruginosa PAO1 has been a biofilm model system in Gram-negative bacteria because of the pathogenic consequences of its attachment to prostheses and its effects in lung colonization. The intermembrane Wsp complex present in $P$. aeruginosa and other pseudomonads modulates attachment controlling the formation of c-di-GMP, the main secondary messenger that regulates the attachment-swimming decision (Simm et al., 2004; Hickman et al., 2005). However, the starting signal that activates the complex is not very well defined yet, although it is probably directly related to physical contact. Amongst the seven protein Wsp intermembrane complex from P. aeruginosa (Figure 1), structural component WspA is usually located 
1 in dynamic clusters in the laterals of bacterial cell surface and, under certain circumstances such as growth on agar surface, it transduces a signal through the entire complex with the special intervention of WspE (Guvener and Harwood, 2007; O'Connor et al., 2012). WspR, another protein in the complex, contains the enzymatic domain GGDEF, able to form c-di-GMP (Simm et al., 2004). Through WspF regulation, WspR gets phosphorylated by WspE and shifts its equilibrium to the formation of c-di-GMP. In the absence of WspF, WspR from P. aeruginosa shows high cyclase activity, increasing c-di-GMP amounts in the cell and leading, consequently, to superior biofilm formation. Therefore, WspF is a regulator by repression of WspR activity (Guvener and Harwood, 2007; Huangyutitham et al., 2013). According to current models, WspR is a cytoplasmic low-activity monomer that changes its conformation upon phophorilation, forming highly-active tetramers (Huangyutitham et al., 2013). Apart from this regulation, WspR activity is repressed by c-di-GMP molecule, which binds to an allosteric site in the GGDEF domain and provokes the dissolution of the clusters and a relaxation of the tetramers leading them to an inhibited WspR dimer form (De et al., 2008; Huangyutitham et al., 2013).

Another c-di-GMP-responsive transcription factor (TF) involved in biofilm formation and motility is the master regulator FleQ, that is also related to other functions, including iron homeostasis (BlancoRomero et al., 2018) and the control of the type 6 secretion system. (T6SS; (Wang et al., 2018)). In $P$. putida in conditions of low c-di-GMP, FleQ can interact with $\sigma^{54}$ to regulate a high number of promoters related to motility and flagella. However, when c-di-GMP concentration is high, this master regulator forms dimers and interacts with $\sigma^{70}$ to activate or repress transcription of biofilm or flagellarelated genes, respectively. In general, c-di-GMP binding to FleQ usually antagonizes its repressor activity, transforming this factor into an activator for the same promoter. This TF is so important in lifestyle decisions that a $P$. putida strain with fle $Q$ deleted resulted in both non-motile and biofilm impaired bacteria (Jimenez-Fernandez et al., 2016; Molina-Henares et al., 2017). FleQ can also interact with another TF, FleN. In P. aeruginosa, FleN acts as an auxiliary element and antagonizes the FleQ dual function activator-repressor for the regulation of certain genes and operons (Dasgupta et al., 2000; Baraquet et al., 2012). In P. putida, FleN and FleQ also usually play opposite roles, with some exceptions such as their synergistic role in regulating transcription of the bcs biofilm operon and lapA adhesin (Nie et al., 2017). 
1 An additional actor of biofilm formation in pseudomonads is FlgZ (also called YcgR in E. coli). This is not a TF but a flagellar function regulator containing a PilZ domain, which can bind c-di-GMP (Amikam and Galperin, 2006). flgZ transcription depends on FleQ and FliA. In E.coli, YcgR regulates swimming velocity based on c-di-GMP levels of the cell (Ryjenkov et al., 2006; Boehm et al., 2010; Martinez-Granero et al., 2014; Baker et al., 2016). In the presence of c-di-GMP, it tends to dimerize and interacts with Mot components of the flagellar stator in addition to the motor and regulates its movement, but, at least in Pseudomonas fluorescens, FlgZ does this independent of c-di-GMP under the conditions tested. However, it does participate in the formation of biofilm mediated by c-di-GMP produced by WspR and degraded by the esterase BifA. In $P$. putida its presence is associated to a reduction of flagellar movement (Martinez-Granero et al., 2014).

On the basis of the above, we studied the wsp orthologous operon in P. putida by making individual and block-deletion mutants, in order to determine the importance of the similarities between this complex and $P$. aeruginosa's and to decide how we could take advantage of its function in order to control biofilm formation. We also studied several mutant phenotypes in three different sole carbon sources, as the nature of them influences extracellular polysaccharide (EPS) building blocks used for biofilm formation (Reeves et al., 1996; Ramos et al., 2001; Wan et al., 2018). The results below not only expose the similarities with the P. aeruginosa's Wsp system of reference, but it also reveals a striking dependence of biofilm formation on growth conditions and metabolic status.

\section{RESULTS AND DISCUSSION}

\section{Modulation of Pseudomonas putida attachment to surfaces through the Wsp complex}

First of all, we searched for a region in $P$. putida's genome that showed homology with the operon of $P$. aeruginosa's wsp. Using the MicroScope platform from GenoScope (Évry, France), we found a set of genes from PP_1488 to PP_1494 loci that showed a significant synteny with the corresponding genes in P. aeruginosa, indicating a potentially shared evolutionary origin (Figure 1b). To study the role of the operon in biofilm formation we made nine clean deletions from ATG to stop codon, as indicated in the Materials and Methods section: one of them comprised the whole operon (wsp) while seven of them were deletions per each ORF ( $w s p A, w s p B, w s p C$, wspD, wspE, wspF, wspR) in order to study their individual effect. Finally, one more double mutant was made for the duo $w s p F w s p$, since this region 
encodes the enzymatic activity and its regulatory protein. With this mutant collection in hand we then checked the phenotype of the different strains in terms of biofilm formation, colony morphology and swimming ability. Focusing on biofilm formation assays of Figure 2a and c, we found some differences with respect to three variables: between mutants, along time and regarding carbon source. As we can see, the general overview showed that carbon regime was an important factor for biofilm build up. Wild type $P$. putida KT2440 (wt) and the rest of the mutants showed a different biofilm profile in media supplemented with 3 types of sugar as a sole carbon source. For example, in glucose or fructose was able to form more biofilm than in citrate. For other side, the deletion mutant for the whole wsp operon produced similar amount of biofilm than wt cultures, indicating that the Wsp complex is keeping the cyclase activity of WspR in a low state in the conditions tested.

Considering time as another variable, we also noticed a general decrease in biofilm at $24 \mathrm{~h}$, something that happened to the $w t$ and to most of the mutants too. But, interestingly, although the wspF mutant formed slightly more biofilm than the rest of the deletions in all carbon sources tested at $6 \mathrm{~h}$ of incubation, those differences noticeably increased at $24 \mathrm{~h}$ due to distinct fluctuations between mutants. For example, in M9 citrate medium, the $w s p F$ mutant doubled biofilm formation from $6 \mathrm{~h}$ to $24 \mathrm{~h}$, while the rest of the mutants decreased it slightly, contributing then to produce a higher difference between this deleted strain and the rest. Regarding M9 fructose medium, the $w s p F$ mutant kept a similar biofilm index from $6 \mathrm{~h}$ to $24 \mathrm{~h}$, whereas the rest of the strains showed drops in production. In M9 glucose medium, this decline was specially intense affecting also to $w s p F$ mutant biofilm levels. Although biofilm fluctuations in time are inherent to P. putida, as wt levels showed, our results indicated that in the absence of WspF, WspR activity was not well regulated, leading to a longer in time c-di-GMP synthesis and biofilm production, but with some particularities depending on the carbon regime. On the contrary, wspR mutant was less able to form biofilm in some of the conditions tested (for example, at 6 $\mathrm{h}$ for glucose and fructose). This is consistent with the hypothesis that the activity of WspR, a protein with a GGDEF domain, is controlled by the phosphorylation regulated by WspF, as occurs in $P$. aeruginosa (Guvener and Harwood, 2007). According to this hypothesis for P. putida, in the absence of WspF, WspR would become phosphorylated at a higher rate by WspE such that it would continuously produce c-di-GMP and biofilm, while in the absence of WspR, the lower amount of c-di-GMP present in the bacteria would make it less able to form biofilm. For other side, the double mutant wspF wspR 
1 showed a similar biofilm production compared to the simple mutant $w s p R$, as the deletion of the cyclase

2 protein has a dominant phenotype over the deletion of its regulator.

4 The interplay Wsp/ c-di-GMP / biofilm formation

5

In sum, although the implication of $w s p F$ and $w s p R$ in biofilm formation through the secondary messenger c-di-GMP was evident, we argue that the differences in biofilm levels between all the mutants when we change the carbon source can be due to the particular regulation of other diguanylate cyclases present in the cytoplasm. Those enzymes could vary its activity or expression depending on cAMP balance, energy, oxidation levels and reduction power directly influenced by the carbon source (Huang et al., 2013; Schirmer, 2016; da Costa Vasconcelos et al., 2017). On the other hand, cyclase activity of WspR would likely differ from that of YedQ, as this protein comes from a different organism and they have different expression levels. This would also contribute to the differences in biofilm production between the $w s p F$ and $w s p R$ mutants and their transformation with the external cyclase and esterase.

\section{Colony morphology and swimming abilities}


2 Colony morphology was observed in M9 agar plates with Congo Red and Coomassie staining for EPS

3 inspection. Congo Red is a dye for extracellular polysaccharides, while Coomassie targets extracellular

4 proteins. As a consequence, a colony with a deeper red colour usually correlates with higher biofilm forming ability. This assay revealed a very low red coloration for P. putida wt in all carbon sources tested, while instead revealing a coarse surface for $w s p F$ mutants in the citrate regime and the reddest colour in fructose as sole carbon source compared to the wt strain (Figure 3a). Although Congo Red and Coomassie staining is a qualitative assay for morphology description, and results do not always completely correlate to quantitative biofilm assays, observations for the $w s p F$ mutant were consistent with those shown in Figure 3. Swimming assays showed minimal halo width differences among mutants except in the case of wspF (Figure 3b), which was less able to move along the soft agar in all the tested carbon sources, forming smaller circles, a finding coherent with the higher amount of c-di-GMP produced by this strain.

Taken together, these observations indicate that the $P$. putida Wsp complex works in a similar way as that of $P$. aeruginosa, i.e. the enzymatic activity of $\mathrm{WspR}$ regulates c-di-GMP levels of the cell under the control of $\mathrm{WspF}$, and therefore also regulates biofilm levels. The influence of the carbon source in biofilm production is also remarkable. Some hypotheses and explanations to those considerations are addressed below.

P. putida wsp complementation with P. aeruginosa's genes

In order to see whether Pseudomonas aeruginosa Wsp proteins were able to complement $P$. putida mutants, we cloned the $w s p$ operon, $w s p F, w s p R$ or $w s p F$ wspR from $P$. aeruginosa PAO1 strain in a pSEVA238 plasmid (Silva-Rocha et al., 2013) under the control of the XylS-Pm expression system. Then, we transformed the deletion mutants of Pseudomonas putida with the new plasmids harbouring $P$. aeruginosa's pertinent gene and checked its phenotype both under induction of the expression system with $2.0 \mathrm{mM}$ of $3 \mathrm{MBz}$ and at the basal level (Figure 4). Our main observation was that wspF from $P$. aeruginosa could complement the deficient $w s p F$ P. putida strain in terms of biofilm formation: the phenotype of the $w s p F^{P A}$ strain showed lower levels of biofilm than the non-complemented wspF, both when the expression of $w s p F^{P A}$ was induced and not induced (except in fructose at 6 hours when 
1 induced, which showed lower levels of biofilm than wild type). This result indicates that low levels of

2 WspF from P. aeruginosa are enough to regulate WspR function from P. putida, as the XylS-Pm

3 expression system has very low leakiness (Calles, B., in preparation). However, the rest of the complementations showed a higher biofilm activity than the $P$. putida $w t$ strain, specially at $24 \mathrm{~h}$ and even when they were not induced under most of the conditions tested. These results let us infer that higher levels of WspR correlate with an increase in the amount of c-di-GMP and therefore biofilm formation as well. The possibility that $P$. aeruginosa $\mathrm{WspR}$ is more active than the corresponding enzyme in P. putida deserves further studies.

In the same work package we also ran colony morphology analysis and swimming ability tests of the complemented strains described in the section above, both under $2.0 \mathrm{mM} 3 \mathrm{MBz}$ induction and without it (Supplementary Figure S1). Results showed that complementation of the wspF mutant strain with $w s p F^{P A}$ restored the wild type phenotype in citrate, and exhibited no important differences between high-expressed and low-expressed $\mathrm{WspF}^{\mathrm{PA}}$. But in the other carbon sources, we observed a slight decrease of swimming ability under glucose and fructose regimes and small differences in colony morphology, when induced and not induced, compared to the wild type. On the contrary, a higher expression of $\mathrm{Wsp}^{\mathrm{PA}}, \mathrm{WspF}^{\mathrm{PA}} \mathrm{WspR}^{\mathrm{PA}}$ and $\mathrm{WspR}^{\mathrm{PA}}$ in their pertinent mutant background presented a higher red staining of EPS and a clear reduction of swimming capacity with haloes reduced to the minimum value possible. Combining these findings with the biofilm assays from Figure 3, it is possible that $P$. aeruginosa Wsp complex keeps a higher level of phosphorylated WspR, letting the c-di-GMP concentration rise and endowing the species with a greater biofilm-forming capacity.

c-di-GMP produced by WspR and lifestyle regulators

Although c-di-GMP implication in biofilm formation is clear, this secondary messenger is also involved in multiple other functions (Romling et al., 2013). Therefore, this molecule has to orchestrate a considerable number of activities through mechanisms other than gross variations in its levels. As data from different studies indicate, c-di-GMP cytoplasmic amounts do not differ homogenously when the appropriate stimulus occurs. This means that general c-di-GMP concentration remains the same, while some local variations are produced. Those confined alterations produce the desired response using specific modulators that respond to heterogeneous variations (Dahlstrom and O'Toole, 2017; Dahlstrom 
et al., 2018). To shed some light on the way that c-di-GMP formed from WspR interacts with certain transcription factors and regulators to form biofilm in P. putida, the same type of experiments were performed using combined deletions of three regulators that respond to c-di-GMP in this context: for one side, we focused on FleQ and FleN, transcription factors that are clearly involved in the regulation of motility and sesility (Baraquet et al., 2012; Jimenez-Fernandez et al., 2016; Blanco-Romero et al., 2018). On a different side, FlgZ, also known as YgcR E. coli, is a protein that harbours a c-di-GMP recognizing PilZ domain, which has been determined as a motility regulator through certain flagellar parts (Martinez-Granero et al., 2014). We checked the biofilm forming phenotype of $f l g Z$, fle $Q$, fleN and fleQ fleN deletions in addition to $w s p F$ flgZ, wspF fleQ, wspF fleN and $w s p F$ fleQ fleN associated deletions (Figure 5). On citrate supplemented media, we found after 6 and $24 \mathrm{~h}$ of incubation that each mutant for fle $Q, f l e N$ and fle $Q$ fleN was very similar to its double mutant fle $Q w s p F$, fleN wspF and fle $Q$ fleN wspF respectively. This meant that the phenotype of fle $Q$ mutant was dominant over wspF, and cdi-GMP formed in this last mutant could not translate to a biofilm production in the absence of fle $Q$ gene. However, double mutant $f l g Z w s p F$ behaved as simple mutant $w s p F$, suggesting that wspF phenotype is dominant over flgZ. This means that c-di-GMP formed by WspR could form similar amounts of biofilm in the presence or the absence of FlgZ.

When we checked the morphology phenotype of the various strains, we observed a common pattern in the fleQ, fleN, fleQ fleN mutants and same deletions in combination with $w s p F$ mutant (Supplementary Figure S2). The high levels of biofilm in the $w s p F$-deleted strain dropped clearly when this mutation was combined with fle $Q$, fleN or fle $Q$ fleN deletions, this last triple mutant being more evidently biofilm deficient at $6 \mathrm{~h}$ of incubation in citrate-supplemented media. However, only fle $Q$ and fle $Q$ fleN (but not fleN) mutants were impaired in swimming. Previous studies showed that FleQ is indispensable for this ability and for biofilm formation (Jimenez-Fernandez et al., 2016), such that a fle $Q$-deficient bacteria are unable to swim or form biofilm independently of the cellular c-di-GMP concentration. Yet, in Congo Red and Coomassie staining, double mutant wspF fleQ had a phenotype more similar to the $w s p F$ mutant than to the fle $Q$ mutant, suggesting a FleQ-independent mechanism for EPS secretion. On the other hand, the $f \lg Z w s p F$ double mutant showed a comparable phenotype to the wspF simple mutant, indicating that c-di-GMP formed by WspR did not affect motility or colony morphology through FlgZ in the conditions tested. 
As can be deduced from the results above, the carbon regime was clearly an influencing factor for final biofilm production. Differences in this production were probably caused by the point of the central metabolism where the carbon skeleton was added. Some previous works have noted the relevance of the kind of carbon source in biofilm formation (Reeves et al., 1996; Ramos et al., 2001; Pemmaraju et al., 2016). In the case of $P$. putida, glucose enters the metabolism and has to go through a special glycolysis that resembles a mix of the Entner-Doudoroff (ED) pathway and the Pentose Phosphate pathway (PP), as it cannot be metabolized through the Tricarboxilic acid cycle (TCA) by the classical EmbdenMeyerhof-Parnas (EMP) pathway (Nikel et al., 2015). This regime led to intermediate levels of biofilm formation. On the other side, the use of fructose as a sole carbon source produced the highest levels of biofilm. Both hexoses can be channeled through ED to the PP for catabolism, but also for gluconeogenesis, which could be related with the higher EPS production of those conditions. Another interesting case is citrate, which can be incorporated directly to the TCA cycle and showed the lowest levels of biofilm staining, suggesting that this molecule was mainly used for catabolism.

\section{Conclusion}

Along this work we have seen Wsp complex in P. putida works in a similar way than it does in $P$. aeruginosa. It regulates c-di-GMP levels of the bacteria through two main components: WspR and WspF. WspR is the protein that harbours the GGDEF domain, which endow it with the capacity to cycle c-di-GMP. WspF is a crucial protein for the regulation of WspR activity. For other side, the Wsp complex from $P$. aeruginosa is so evolutionary close to $P$. putida's that $\mathrm{WspF}^{\mathrm{PA}}$ can modulate $\mathrm{WspR}^{\mathrm{PP}}$ phosphorylation by $\mathrm{WspE}^{\mathrm{PP}}$ and slow down its cyclase activity, complementing the function of $\mathrm{WspF}^{\mathrm{PP}}$ under certain conditions. The rest of complementations with $P$. aeruginosa's genes showed different biofilm and swimming activities to its respective mutants, and although the wild type phenotype could not be rescued the reason was probably related to expression dose of the Wsp proteins or to a higher cyclase activity of $\mathrm{WspR}^{\mathrm{PA}}$ compared to $\mathrm{WspR}^{\mathrm{PP}}$. Further enzymatic activity studies should be done in order to test this last hypothesis. Regarding c-di-GMP effector proteins, transcription factor FleQ is a key regulator for biofilm production and swimming abilities of $P$. putida, so its deletion phenotype will be always dominant over other deletions of c-di-GMP forming or degrading proteins. Therefore, fle $Q$ 
mutant and double mutant strains are impaired in those activities independently of the c-di-GMP levels. Finally, the considerable influence of carbon source on biofilm formation was clearly evident: $P$. putida and most of the mutants showed higher biofilm production levels with fructose as the only carbon source, while exhibiting an intermediate biofilm production in glucose and the least biofilm amounts in our experiments in citrate cultures. We consider the information obtained by those experiments is of relevance for further understanding of biofilm formation in $P$. putida and its possible control with multiple applications.

\section{EXPERIMENTAL PROCEDURES}

Strains, culture conditions and plasmid construction

Bacterial strains used in this study are listed in Table S1. All P. putida mutants were derived from the reference wild type strain KT2440 (Nelson et al., 2002). For the construction of new plasmids, E. coli strains DH5 $\alpha$, CC118, JM109, and DH5 $\alpha$ ppir were used. The last strain is necessary for the replication of R6K origin plasmids (as pEMG) because it encodes the $\pi$ replication protein. Pseudomonas aeruginosa genes were cloned by PCR amplification using PAO1 strain as template. For general methods, Escherichia coli and Pseudomonas putida strains were cultured in Luria Bertani (LB) medium, while for the specific biofilm, swimming and colony morphology assays, M9 minimal medium supplemented with citrate $0.2 \%,(\mathrm{w} / \mathrm{v})$, glucose $0.2 \%(\mathrm{w} / \mathrm{v})$ or fructose $0.2 \%(\mathrm{w} / \mathrm{v})$ was used. A temperature of $37^{\circ} \mathrm{C}$ was set for $E$. coli and $P$. aeruginosa cultures and of $30^{\circ} \mathrm{C}$ for $P$. putida. Solid plates were made with the same media adding $1.5 \%(\mathrm{w} / \mathrm{v})$ for general incubations, while a concentration of agar at $0.3 \%(\mathrm{w} / \mathrm{v})$ was utilized for swimming assays. Antibiotics were added at the proper concentrations when it was necessary: kanamycin $\left(\mathrm{Km}, 50 \mu \mathrm{g} \mathrm{mL} L^{-1}\right)$, ampicillin $\left(\mathrm{Ap}, 150 \mu \mathrm{g} \mathrm{mL} \mathrm{L}^{-1}\right.$ for $E$. coli and $500 \mu \mathrm{g} \mathrm{mL}^{-1}$ for $P$. putida) and tetracycline (Tc, $10 \mu \mathrm{g} \mathrm{mL} \mathrm{m}^{-1}$ for E. coli and $5 \mu \mathrm{g} \mathrm{mL}^{-1}$ for $P$. putida). When needed, cultures were induced with the chemical 3-methilbenzoate (3MBz), also known as $m$-toluate (Sigma Aldrich, St Louis, Missouri, USA) at $2 \mathrm{mM}$. Plasmids built and employed in this work are listed in Table S2. Classical protocols of digestion, ligation and transformation for E. coli were used for the most of the constructs (Maniatis, 1982). PCRs were performed using Q5 High Fidelity polymerase or Phusion High Fidelity polymerase (both from New England Biolabs, Ipswich, Massachusetts, USA), and analytic PCRs were performed using MasterMix (Promega, Madison, 
1 Wisconsin, USA) or Green MasterMix (BioTools, Madrid, Spain). For digestions, restriction enzymes 2 (New England Biolabs, Ipswich, Massachusetts, USA) were used and for ligations, T4 ligase (Roche, 3 Basilea, Switzerland) was ordered. Plasmid DNA extraction from bacteria was performed using 4 QIAprep Spin Miniprep Kit (Qiagen, Venlo, Netherlands). DNA was isolated from agarose bands with 5 the NucleoSpin Extract II kit (MN, Düren, Germany). E. coli strains were either heat-shocked (90 6 seconds at $42{ }^{\circ} \mathrm{C}$ ) or electro-shocked (cuvettes $0.2 \mathrm{~cm}, 2.4 \mathrm{kv}$ ) for transformations. For the 7 transformations and deletions in P. putida, protocols for Gram-negative bacteria described by Martinez8 Garcia and de Lorenzo (2011) were used. For the clean deletions, pEMG plasmids (listed in 9 Supplementary Table S2) were built with the 500 bp's flanking regions upstream and downstream of the 10 genes to be deleted. Primers used for the amplification of this homology regions can be found in 11 Supplementary Table S3.

Crystal Violet assays

The protocol described by O'Toole and Kolter (1998) was used to perform Crystal Violet experiments. Different $P$. putida mutant strains were cultured overnight $(\mathrm{O} / \mathrm{N})$ at $30^{\circ} \mathrm{C}$ in the medium of interest. The following morning, transparent 96-well plates with flat bottoms were inoculated using $0.2 \mu \mathrm{L}$ of $\mathrm{O} / \mathrm{N}$ preinocula in $200 \mu \mathrm{L}$ of the same medium. The plates were placed at $30^{\circ} \mathrm{C}$ without shaking during 6 or $24 \mathrm{~h}$, and then $\mathrm{OD}_{600}$ was measured again and the biofilm stained with Crystal Violet at $1 \%(\mathrm{w} / \mathrm{v})$ and later dissolved with acetic acid 33\% (w/v). Crystal Violet intensity was measured at $\mathrm{OD}_{595}$. Both $\mathrm{OD}_{600}$ and $\mathrm{OD}_{595}$ were measured using SpectraMax iD3 (Molecular Devices, California, USA) plate reader. Biofilm index was calculated by dividing both measurements $\mathrm{OD}_{595} / \mathrm{OD}_{600}$ and the resulting value was plotted.

Swimming assays and Congo Red Coomassie tests

For the swimming experiments, M9 medium supplemented with the different carbon sources and $0.3 \%$ (w/v) agar was used. Overnight cultures grown in M9 supplemented with the corresponding carbon source were diluted to an $\mathrm{OD}_{600}$ of 0.5 . Aliquots of $3 \mu \mathrm{L}$ of every dilution were placed onto soft agar plates with the same medium. Pictures of the swimming halos were taken after $24 \mathrm{~h}$ of incubation at $30^{\circ} \mathrm{C}$. Colony morphology of the different strains was studied adding $40 \mu \mathrm{g} / \mu \mathrm{L}$ of Congo Red and 20 
$\mu \mathrm{g} / \mu \mathrm{L}$ of Coomassie brilliant Blue on different M9 media. Overnight cultures were adjusted to $\mathrm{OD}_{600}$ of 0.5 , and a $3 \mu \mathrm{L}$ drop was placed on the top of the agar. The plates were incubated at $30^{\circ} \mathrm{C}$ during 7 days, following which pictures of the colonies were taken.

\section{ACKNOWLEDGEMENTS}

This work was funded by the SETH Project of the Spanish Ministry of Science RTI 2018-095584-BC42, MADONNA (H2020-FET-OPEN-RIA-2017-1-766975), BioRoboost (H2020-NMBP-BIO-CSA2018), and SYNBIO4FLAV (H2020-NMBP/0500) Contracts of the European Union and the S2017/BMD-3691 InGEMICS-CM funded by the Comunidad de Madrid (European Structural and Investment Funds). Authors declare no conflict of interests.

\section{SUPPLEMENTARY MATERIALS}

Supplementary Table S1: Escherichia coli and Pseudomonas strains used in this work

Supplementary Table S2: Plasmids built and used in this work

Supplementary Table S3: Primers designed for $w s p$ experiments

Supplementary Figure S1: Colony morphology and swimming ability for $P$. putida wsp mutants and their complementation with orthologous genes from $P$. aeruginosa

Supplementary Figure S2: Colony morphology and swimming ability of strains lacking transcription factors FleQ and FleN and for the regulator FlgZ, associated to wspF mutant

\section{REFERENCES}

Amikam, D., and Galperin, M.Y. (2006) PilZ domain is part of the bacterial c-di-GMP binding protein. Bioinformatics 22: 3-6.

Baker, A.E., Diepold, A., Kuchma, S.L., Scott, J.E., Ha, D.G., Orazi, G. et al. (2016) PilZ Domain Protein FlgZ Mediates Cyclic Di-GMP-Dependent Swarming Motility Control in Pseudomonas aeruginosa. J Bacteriol 198: 1837-1846.

Baraquet, C., Murakami, K., Parsek, M.R., and Harwood, C.S. (2012) The FleQ protein from Pseudomonas aeruginosa functions as both a repressor and an activator to control gene expression from the pel operon promoter in response to c-di-GMP. Nucleic Acids Res 40: 72077218. 
Blanco-Romero, E., Redondo-Nieto, M., Martinez-Granero, F., Garrido-Sanz, D., Ramos-Gonzalez, M.I., Martin, M., and Rivilla, R. (2018) Genome-wide analysis of the FleQ direct regulon in Pseudomonas fluorescens F113 and Pseudomonas putida KT2440. Sci Rep 8: 018-31371.

Boehm, A., Kaiser, M., Li, H., Spangler, C., Kasper, C.A., Ackermann, M. et al. (2010) Second messenger-mediated adjustment of bacterial swimming velocity. Cell 141: 107-116.

da Costa Vasconcelos, F.N., Maciel, N.K., Favaro, D.C., de Oliveira, L.C., Barbosa, A.S., Salinas, R.K. et al. (2017) Structural and Enzymatic Characterization of a cAMP-Dependent Diguanylate Cyclase from Pathogenic Leptospira Species. J Mol Biol 429: 2337-2352.

Dahlstrom, K.M., and O'Toole, G.A. (2017) A Symphony of Cyclases: Specificity in Diguanylate Cyclase Signaling. Annu Rev Microbiol 71: 179-195.

Dahlstrom, K.M., Collins, A.J., Doing, G., Taroni, J.N., Gauvin, T.J., Greene, C.S. et al. (2018) A Multimodal Strategy Used by a Large c-di-GMP Network. J Bacteriol 200: 00703-00717.

Dasgupta, N., Arora, S.K., and Ramphal, R. (2000) fleN, a gene that regulates flagellar number in Pseudomonas aeruginosa. J Bacteriol 182: 357-364.

De, N., Pirruccello, M., Krasteva, P.V., Bae, N., Raghavan, R.V., and Sondermann, H. (2008) Phosphorylation-independent regulation of the diguanylate cyclase WspR. PLoS Biol 6: 0060067.

Guvener, Z.T., and Harwood, C.S. (2007) Subcellular location characteristics of the Pseudomonas aeruginosa GGDEF protein, WspR, indicate that it produces cyclic-di-GMP in response to growth on surfaces. Mol Microbiol 66: 1459-1473.

Hickman, J.W., Tifrea, D.F., and Harwood, C.S. (2005) A chemosensory system that regulates biofilm formation through modulation of cyclic diguanylate levels. Proc Natl Acad Sci U S A 102: $14422-14427$.

Huang, C.J., Wang, Z.C., Huang, H.Y., Huang, H.D., and Peng, H.L. (2013) YjcC, a c-di-GMP phosphodiesterase protein, regulates the oxidative stress response and virulence of Klebsiella pneumoniae CG43. PLoS One 8.

Huangyutitham, V., Guvener, Z.T., and Harwood, C.S. (2013) Subcellular clustering of the phosphorylated WspR response regulator protein stimulates its diguanylate cyclase activity. MBio 4: 00242-00213.

Jimenez-Fernandez, A., Lopez-Sanchez, A., Jimenez-Diaz, L., Navarrete, B., Calero, P., Platero, A.I., and Govantes, F. (2016) Complex Interplay between FleQ, Cyclic Diguanylate and Multiple sigma Factors Coordinately Regulates Flagellar Motility and Biofilm Development in Pseudomonas putida. PLoS One 11.

Maniatis, F.a.S. (1982) Molecular cloning: a laboratory manual In: Cold Spring Harbor Laboratory Press. 
Martinez-Garcia, E., and de Lorenzo, V. (2011) Engineering multiple genomic deletions in Gramnegative bacteria: analysis of the multi-resistant antibiotic profile of Pseudomonas putida KT2440. Environ Microbiol 13: 2702-2716.

Martinez-Granero, F., Navazo, A., Barahona, E., Redondo-Nieto, M., Gonzalez de Heredia, E., Baena, I. et al. (2014) Identification of flgZ as a flagellar gene encoding a PilZ domain protein that regulates swimming motility and biofilm formation in Pseudomonas. PLoS One 9.

Molina-Henares, M.A., Ramos-Gonzalez, M.I., Daddaoua, A., Fernandez-Escamilla, A.M., and Espinosa-Urgel, M. (2017) FleQ of Pseudomonas putida KT2440 is a multimeric cyclic diguanylate binding protein that differentially regulates expression of biofilm matrix components. Res Microbiol 168: 36-45.

Nie, H., Xiao, Y., Liu, H., He, J., Chen, W., and Huang, Q. (2017) FleN and FleQ play a synergistic role in regulating lapA and bcs operons in Pseudomonas putida KT2440. Environ Microbiol Rep 9: 571-580.

Nikel, P.I., Chavarria, M., Fuhrer, T., Sauer, U., and de Lorenzo, V. (2015) Pseudomonas putida KT2440 Strain Metabolizes Glucose through a Cycle Formed by Enzymes of the EntnerDoudoroff, Embden-Meyerhof-Parnas, and Pentose Phosphate Pathways. J Biol Chem 290: 25920-25932.

O'Connor, J.R., Kuwada, N.J., Huangyutitham, V., Wiggins, P.A., and Harwood, C.S. (2012) Surface sensing and lateral subcellular localization of $\mathrm{WspA}$, the receptor in a chemosensory-like system leading to c-di-GMP production. Mol Microbiol 86: 720-729.

O'Toole, G.A., and Kolter, R. (1998) Flagellar and twitching motility are necessary for Pseudomonas aeruginosa biofilm development. Mol Microbiol 30: 295-304.

Pemmaraju, S.C., Pruthi, P.A., Prasad, R., and Pruthi, V. (2016) Modulation of Candida albicans Biofilm by Different Carbon Sources. Mycopathologia 181: 341-352.

Ramos, A., Boels, I.C., de Vos, W.M., and Santos, H. (2001) Relationship between glycolysis and exopolysaccharide biosynthesis in Lactococcus lactis. Appl Environ Microbiol 67: 33-41.

Reeves, P.R., Hobbs, M., Valvano, M.A., Skurnik, M., Whitfield, C., Coplin, D. et al. (1996) Bacterial polysaccharide synthesis and gene nomenclature. Trends Microbiol 4: 495-503.

Romling, U., Galperin, M.Y., and Gomelsky, M. (2013) Cyclic di-GMP: the first 25 years of a universal bacterial second messenger. Microbiol Mol Biol Rev 77: 1-52.

Ryjenkov, D.A., Simm, R., Romling, U., and Gomelsky, M. (2006) The PilZ domain is a receptor for the second messenger c-di-GMP: the PilZ domain protein YcgR controls motility in enterobacteria. J Biol Chem 281: 30310-30314.

Schirmer, T. (2016) C-di-GMP Synthesis: Structural Aspects of Evolution, Catalysis and Regulation. $J$ Mol Biol 428: 3683-3701. 
1 Silva-Rocha, R., Martinez-Garcia, E., Calles, B., Chavarria, M., Arce-Rodriguez, A., de Las Heras, A. et al. (2013) The Standard European Vector Architecture (SEVA): a coherent platform for the analysis and deployment of complex prokaryotic phenotypes. Nucleic Acids Res 41: 23.

Simm, R., Morr, M., Kader, A., Nimtz, M., and Romling, U. (2004) GGDEF and EAL domains inversely regulate cyclic di-GMP levels and transition from sessility to motility. Mol Microbiol 53: $1123-1134$.

Wan, N., Wang, H., Ng, C.K., Mukherjee, M., Ren, D., Cao, B., and Tang, Y.J. (2018) Bacterial Metabolism During Biofilm Growth Investigated by (13)C Tracing. Front Microbiol 9.

Wang, Y., Li, Y., Wang, J., and Wang, X. (2018) FleQ regulates both the type VI secretion system and flagella in Pseudomonas putida. Biotechnol Appl Biochem 65: 419-427. 
1

2

3

4

5

6

7

8

9

\section{FIGURES}

Figure 1: Wsp complex in Pseudomonas sp.

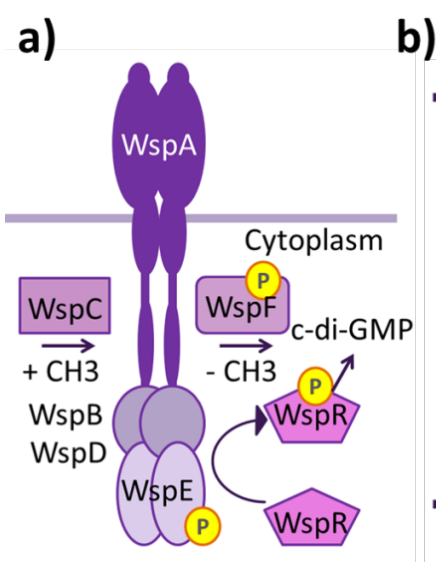

b)

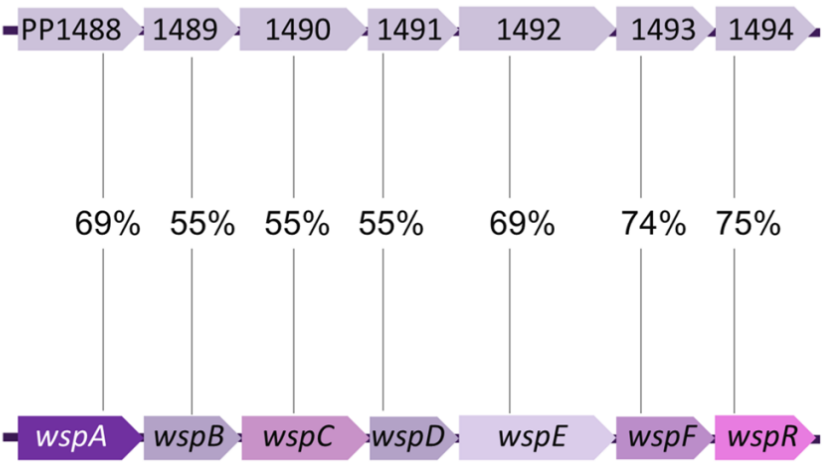

(a) Model proposed for the structure of Wsp protein complex from P. aeruginosa (Guvener and Harwood, 2007). P. putida's complex probably shows the same disposition. (b) Comparison between the seven genes of Wsp operon form $P$. aeruginosa (lower panel) and the putative syntenic operon found in $P$ putida (upper panel). Identity percentage per gene is shown. 
2 Figure 2: Biofilm assay for P. putida wsp mutants and their transformations with YedQ and YljH.

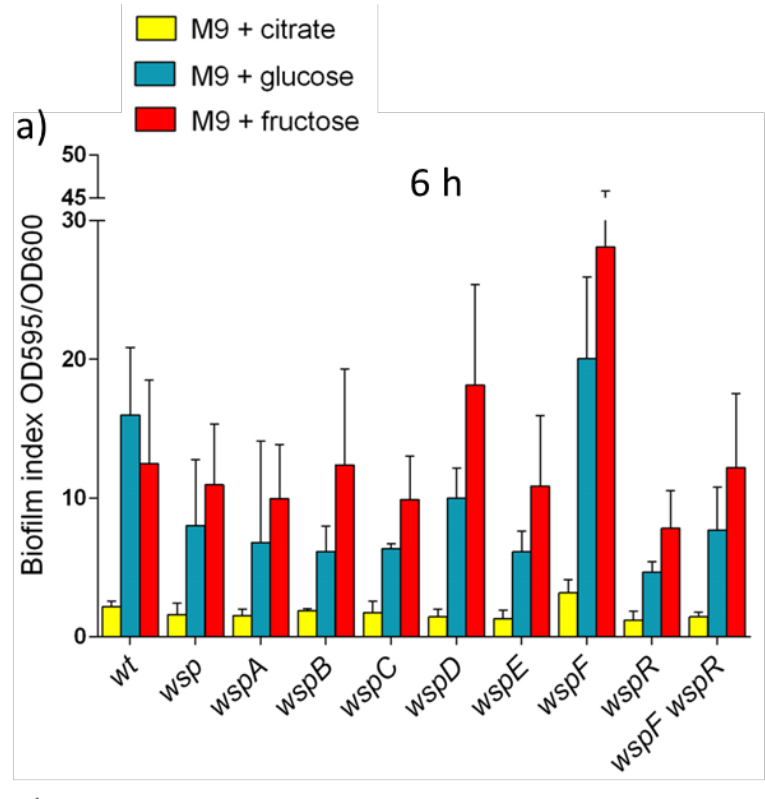

c)

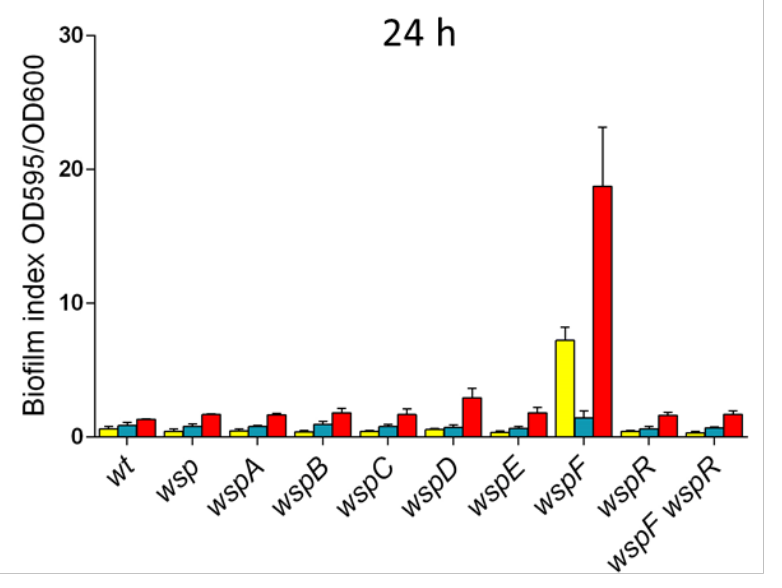

b)

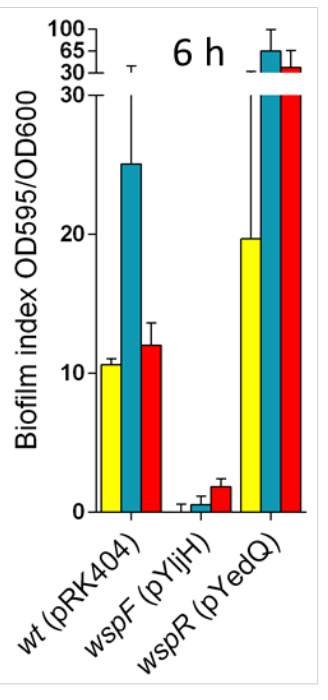

d)

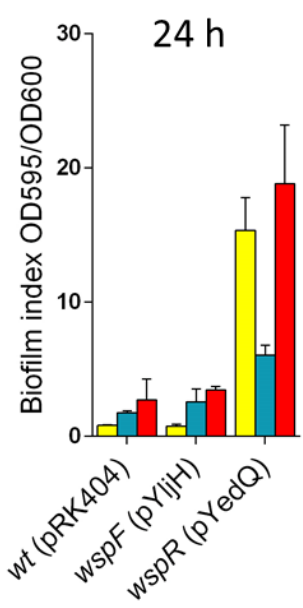

5 
2 Figure 3: Colony morphology and swimming assay for P. putida wsp mutants.

a)
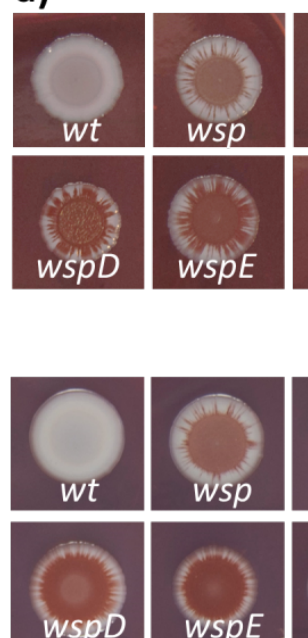

WspD

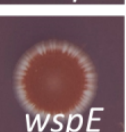

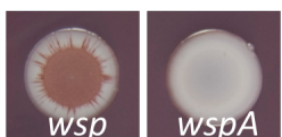
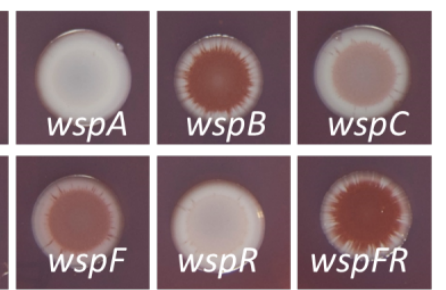

$\mathrm{M} 9$ glucose
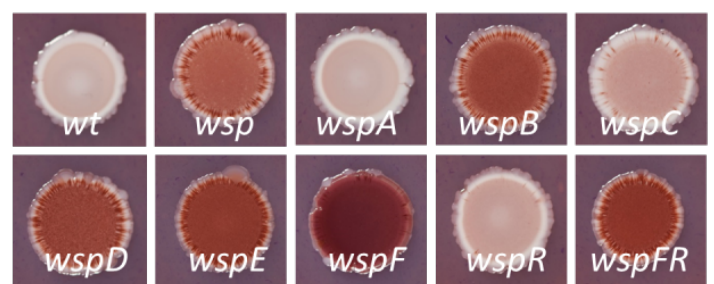

M9 fructose

b)
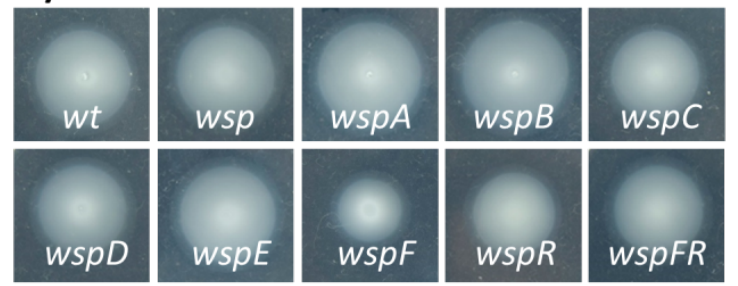

M9 citrate
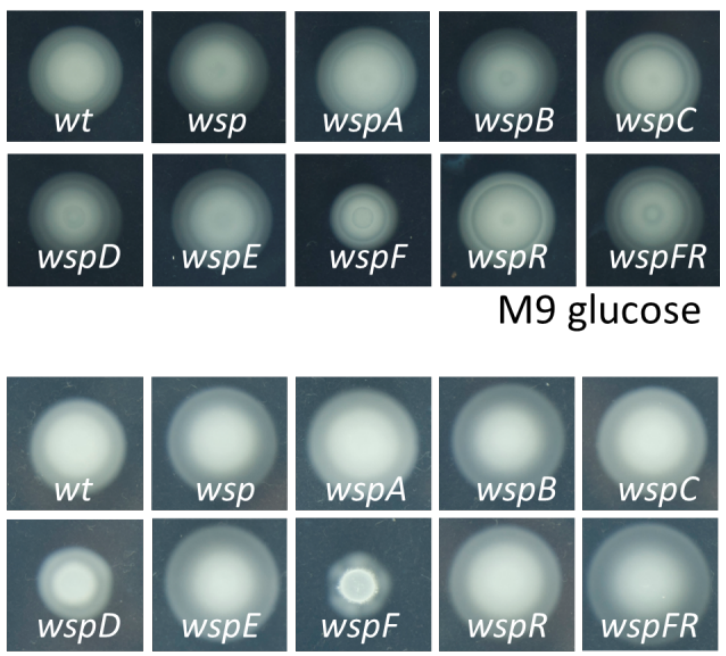

M9 fructose

\section{(}

(a) EPS inspection of wsp mutants was carried out using Congo Red/Coomassie staining for M9 solid media supplemented with citrate, glucose or fructose. Colony colour intensity correlates with the amount 8 of produced biofilm. (b) Swimming assays were performed in M9 $0.3 \%$ agar (w/v) media supplemented 9 with citrate, glucose or fructose as sole carbon sources. The $w s p F$ mutant was less able to swim in all of 0 the carbon sources tested. 
$3 \quad$ Figure 4: Biofilm assay for P. putida wsp mutants and their complementations with the orthologous 4 genes from $P$. aeruginosa.

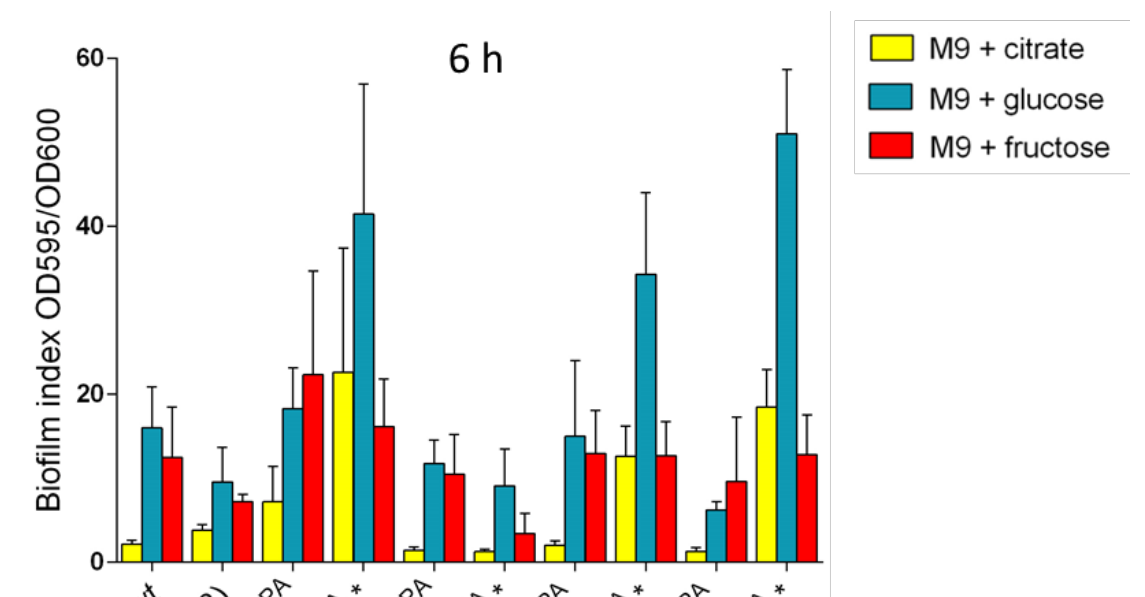

8 The experiment was performed in M9 supplemented with citrate (yellow), glucose (blue) or fructose 9 (red) media. P. putida's $w s p, w s p F, w s p R$ and $w s p F$ wspR mutants were transformed with pSEVA238 10 harbouring genes $w s p\left(w s p^{\mathrm{PA}}\right), w s p F\left(w s p F^{\mathrm{PA}}\right), w s p R\left(w s p R^{\mathrm{PA}}\right)$ and $w s p F w s p R\left(w s p F^{\mathrm{PA}} w s p R^{\mathrm{PA}}\right)$ from $11 P$. aeruginosa respectively. $P$. putida $w t$ was also transformed with the empty vector pSEVA238. The 12 expression of complemented genes was analysed under non-inducing conditions or in the presence of 2 $\mathrm{mM} 3 \mathrm{MBz}$ as inducer of the system (indicated with an asterisk), in order to check the activity of $P$. 
$1 \quad$ Figure 5: Biofilm assay of $P$. putida mutant FleQ, FleN and FlgZ in relation to WspF deficiency.

5 Cultures of each of the mutants indicated were incubated in M9 medium supplemented with citrate 6 (yellow), glucose (blue) and fructose (red) as sole carbon source during $6 \mathrm{~h}$ and $24 \mathrm{~h}$. Simple deletions 7 (wspF, flgZ, fleQ and fleN) were compared to double and triple mutants (fleQ fleN, flgZ wspF, fle $Q$ $8 w s p F$, fleN $w s p F$ and fle $Q$ fleN $w s p F$ ) in order to find epistatic relations.
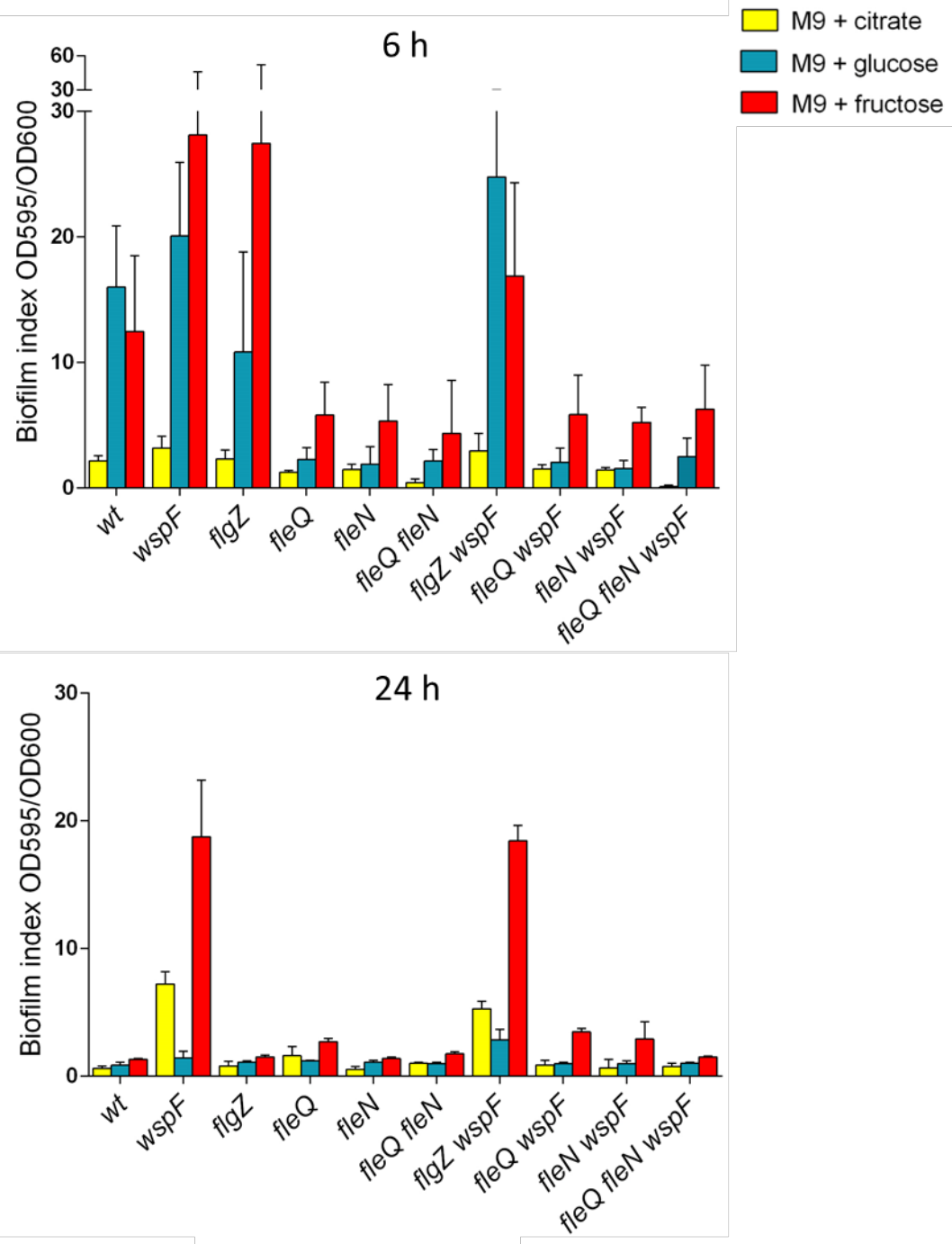